\title{
Mimetic dark matter, ghost instability and a mimetic tensor-vector-scalar gravity
}

\author{
Masud Chaichian, ${ }^{a}$ Josef Klusoň, ${ }^{b}$ Markku Oksanen ${ }^{a}$ and Anca Tureanu ${ }^{a}$ \\ ${ }^{a}$ Department of Physics, University of Helsinki, \\ P.O. Box 64, FI-00014 Helsinki, Finland \\ ${ }^{b}$ Department of Theoretical Physics and Astrophysics, \\ Faculty of Science, Masaryk University, \\ Kotlářská 2, 611 37, Brno, Czech Republic \\ E-mail: masud.chaichian@helsinki.fi, klu@physics.muni.cz, \\ markku.oksanen@helsinki.fi, anca.tureanu@helsinki.fi
}

ABSTRACT: Recently modified gravitational theories which mimic the behaviour of dark matter, the so-called "Mimetic Dark Matter", have been proposed. We study the consistency of such theories with respect to the absence of ghost instability and propose a new tensor-vector-scalar theory of gravity, which is a generalization of the previous models of mimetic dark matter with additional desirable features. The original model proposed by Chamseddine and Mukhanov [JHEP 11 (2013) 135] is concluded to describe a regular pressureless dust, presuming that we consider only those configurations where the energy density of the mimetic dust remains positive under time evolution. For certain type of configurations the theory can become unstable. Both alternative modified theories of gravity, which are based on a vector field (tensor-vector theory) or a vector field and a scalar field (tensor-vector-scalar theory), are free of ghost instabilities.

KeYwords: Cosmology of Theories beyond the SM, Classical Theories of Gravity

ARXiv EPRINT: 1404.4008 


\section{Contents}

1 Introduction 1

2 Hamiltonian analysis of mimetic dark matter model 2

$\begin{array}{ll}2.1 \text { Gauge fixing of scale symmetry } & 10\end{array}$

2.2 Dust structure of the Hamiltonian 11

3 Mimetric theory in Einstein frame 12

4 Vector field model of mimetic dark matter 12

5 Mimetic tensor-vector-scalar gravity $\quad \mathbf{1 5}$

$\begin{array}{ll}5.1 \text { Hamiltonian formulation } & 17\end{array}$

$\begin{array}{llr}6 & \text { Conclusions } & 19\end{array}$

\section{Introduction}

Recently a new interesting model of mimetic dark matter was suggested in [1] and was further elaborated in $[2,3]$. The basic idea is remarkably simple. The physical metric $g_{\mu \nu}^{\text {phys }}$ is considered to be a function of a scalar field $\phi$ and a fundamental metric $g_{\mu \nu}$, where the physical metric is defined as ${ }^{1}$

$$
g_{\mu \nu}^{\text {phys }}=\left(-g^{\alpha \beta} \partial_{\alpha} \phi \partial_{\beta} \phi\right) g_{\mu \nu} .
$$

The physical metric $g_{\mu \nu}^{\text {phys }}$ is invariant with respect to the Weyl transformation of the metric $g_{\mu \nu}$,

$$
g_{\mu \nu}^{\prime}(x)=\Omega^{2}(x) g_{\mu \nu}(x) .
$$

Then it was shown in [1] and in [2] that the ordinary Einstein-Hilbert action constructed using the physical metric $g_{\mu \nu}^{\text {phys }}$ possesses many interesting properties. In fact, the model analyzed below is a conformal extension of Einstein's general theory of relativity. The local Weyl invariance is ensured by introducing an extra degree of freedom that as was shown in [1] has the form of pressureless perfect fluid that, according to [1], can mimic the behavior of a real cold dark matter.

Historically, Gunnar Nordström was the first to construct a relativistic theory of gravity as a scalar field theory [4] whose geometric reformulation [5] was the first metric theory of gravity. The physical metric of this gravitational theory was defined as a conformal transformation of the flat Minkowski metric, $g_{\mu \nu}=\phi^{2} \eta_{\mu \nu}$, where $\phi$ is the scalar field of Nordström's theory. In other words, it was a theory of conformally flat spacetimes. The structure of the field equation, $R=24 \pi G T$, where $R$ and $T$ are the traces of the Ricci tensor and the energy-momentum tensor respectively, closely resembled the field equation of the general theory of relativity formulated by Einstein in the following year. Intriguingly,

\footnotetext{
${ }^{1}$ We follow the convention used in [2] and we also consider the space-time metric of the signature $(-,+,+,+)$.
} 
the idea of mimetic matter [1] is to introduce additional fields in the conformal factor that relates the physical and auxiliary metrics (1.1) in such a way that the physical metric remains invariant under the conformal transformation (1.2).

The mimetic dark matter proposal [1] is very interesting and certainly deserves further study. In general, formulation of the theory using $g_{\mu \nu}^{\text {phys }}$ can lead to a theory with higher order derivatives of $\phi$, which may imply the emergence of ghosts. In order to answer this question, it is necessary to obtain the Hamiltonian formulation of given theory, identify all constraints and perform the counting of all local degrees of freedom. A preliminary analysis of the ghost issue was performed in [2], where however the gauge fixing condition $g^{\mu \nu} \partial_{\mu} \phi \partial_{\nu} \phi+1=0$ was imposed before proceeding to the canonical formulation. We rather start with the original action and perform its canonical analysis in full generality. First we rewrite the action into a form that does not contain derivatives higher than the first order. Surprisingly we find that the action resembles the Lagrange multiplier modified action [6] (see also [7, 8]) whose Hamiltonian analysis was performed in [9]. By solving the second class constraints we derive the Hamiltonian for the scalar field, which turns out to be linear in the momentum conjugate to the scalar field. Since this kind of Hamiltonians often are unstable, especially in higher derivative theories, a careful analysis is required. The Hamiltonian of the original model [1] is argued to be unbounded from below for certain type of initial configurations and consequently it can become unstable. Then we perform the Hamiltonian analysis of the Proca vector field model suggested in [2]. Since now there are no derivatives higher than the first order, the Hamiltonian constraint is found to depend quadratically on the momenta conjugate to the vector field so that it is bounded from below. This is a very interesting result that implies that the Proca model should be studied further. Finally, we present a mimetic tensor-vector-scalar gravity which is a generalization of the previous models [1-3] featuring mimetic matter, and that shares some properties with the celebrated tensor-vector-scalar theories of gravity proposed by Bekenstein [10] and Moffat [11]. It has been recently shown that Bekenstein's tensorvector-scalar gravity is free of ghost degrees of freedom provided that its scalar and vector fields satisfy a certain condition [12].

The organization of this paper is as follows. In section 2 , we introduce the mimetic dark matter action that was suggested in [1]. Then we perform its canonical analysis. We identify all constraints and determine the number of physical degrees of freedom. Gauge fixing of the conformal symmetry and the dust structure of the Hamiltonian are discussed in sections 2.1 and 2.2. In section 3, we discuss the formulation of the theory in the Einstein frame. In section 4, we perform the canonical analysis of the Proca model introduced in [2]. In section 5, we propose a tensor-vector-scalar theory that is a generalization of the aforementioned models of mimetic dark energy.

\section{Hamiltonian analysis of mimetic dark matter model}

In this section we perform the Hamiltonian analysis of the mimetic dark matter model that was introduced in [1]. The gravitational action is defined as

$$
S\left[g_{\mu \nu}, \phi\right]=\frac{1}{2} \int d^{4} x \sqrt{-g^{\text {phys }}\left(g_{\mu \nu}, \phi\right)} R\left(g_{\mu \nu}^{\text {phys }}\left(g_{\mu \nu}, \phi\right)\right),
$$


where we have set $8 \pi G=1$ and where the physical metric is parameterized in terms of the fundamental metric $g_{\mu \nu}$ and the space-time gradients of the scalar field $\phi$ as

$$
g_{\mu \nu}^{\text {phys }}=\left(-g^{\alpha \beta} \partial_{\alpha} \phi \partial_{\beta} \phi\right) g_{\mu \nu} \equiv \Phi^{2} g_{\mu \nu} .
$$

Matter fields couple to the physical metric minimally. We omit matter fields in our analysis since their contribution is similar as in general relativity. To proceed further we express the action using the metric $g_{\mu \nu}$ rather than the physical metric $g_{\mu \nu}^{\text {phys }}$. This can be done using the well known relation, see for example [13],

$$
R\left(g_{\mu \nu}^{\text {phys }}\right)=\frac{1}{\Phi^{2}}\left(R\left(g_{\mu \nu}\right)-6 \frac{g^{\mu \nu} \nabla_{\mu} \nabla_{\nu} \Phi}{\Phi}\right)
$$

where the covariant derivative $\nabla_{\mu}$ is defined using the metric $g_{\mu \nu}$. Inserting (2.3) into (2.1) we obtain

$$
S\left[g_{\mu \nu}, \phi\right]=\frac{1}{2} \int d^{4} x \sqrt{-g}\left[\Phi^{2} R\left(g_{\mu \nu}\right)+6 g^{\mu \nu} \nabla_{\mu} \Phi \nabla_{\nu} \Phi\right] .
$$

Clearly the action is invariant under the conformal transformation (1.2) of the metric $g_{\mu \nu}$, since the metric $g_{\mu \nu}^{\text {phys }}$ is invariant by construction. On the other hand we see that this action contains second order derivative of $\phi$ so that we should worry about possible existence of the ghosts. In order to obtain an action with the first order derivatives, we introduce an auxiliary field $\lambda$ and rewrite the action (2.4) into the form

$$
S\left[g_{\mu \nu}, \Phi, \lambda, \phi\right]=\frac{1}{2} \int d^{4} x \sqrt{-g}\left[R\left(g_{\mu \nu}\right) \Phi^{2}+6 g^{\mu \nu} \nabla_{\mu} \Phi \nabla_{\nu} \Phi-\lambda\left(\Phi^{2}+g^{\mu \nu} \nabla_{\mu} \phi \nabla_{\nu} \phi\right)\right],
$$

where now we treat $\Phi$ as an independent field together with $\phi$. Note that solving the equation of motion for $\lambda$ we find $\Phi^{2}=-g^{\mu \nu} \nabla_{\mu} \phi \nabla_{\nu} \phi$. Then inserting this result into (2.5) we can derive the original action (2.4).

Now we can proceed to the Hamiltonian formulation of the theory. We use the following $3+1$ decomposition of the metric $g_{\mu \nu}[14,15]$,

$$
\begin{aligned}
& g_{00}=-N^{2}+N_{i} h^{i j} N_{j}, \quad g_{0 i}=N_{i}, \quad g_{i j}=h_{i j}, \\
& g^{00}=-\frac{1}{N^{2}}, \quad g^{0 i}=\frac{N^{i}}{N^{2}}, \quad g^{i j}=h^{i j}-\frac{N^{i} N^{j}}{N^{2}},
\end{aligned}
$$

where we have defined $h^{i j}$ as the inverse to the induced metric $h_{i j}$ on the Cauchy surface $\Sigma_{t}$ at each time $t$

$$
h_{i k} h^{k j}=\delta_{i}{ }^{j},
$$

and we denote $N^{i}=h^{i j} N_{j}$. The four dimensional scalar curvature in $3+1$ formalism has the form

$$
R\left(g_{\mu \nu}\right)=K_{i j} \mathcal{G}^{i j k l} K_{k l}+R+\frac{2}{\sqrt{-g}} \partial_{\mu}\left(\sqrt{-g} n^{\mu} K\right)-\frac{2}{\sqrt{h} N} \partial_{i}\left(\sqrt{h} h^{i j} \partial_{j} N\right),
$$

where the extrinsic curvature of the spatial hypersurface $\Sigma_{t}$ at time $t$ is defined as

$$
K_{i j}=\frac{1}{2 N}\left(\frac{\partial h_{i j}}{\partial t}-D_{i} N_{j}-D_{j} N_{i}\right),
$$


with $D_{i}$ being the covariant derivative determined by the metric $h_{i j}$, and where the de Witt metric is defined as

$$
\mathcal{G}^{i j k l}=\frac{1}{2}\left(h^{i k} h^{j l}+h^{i l} h^{j k}\right)-h^{i j} h^{k l}
$$

with inverse

$$
\mathcal{G}_{i j k l}=\frac{1}{2}\left(h_{i k} h_{j l}+h_{i l} h_{j k}\right)-\frac{1}{2} h_{i j} h_{k l}
$$

that obeys the relation

$$
\mathcal{G}_{i j k l} \mathcal{G}^{k l m n}=\frac{1}{2}\left(\delta_{i}^{m} \delta_{j}^{n}+\delta_{i}^{n} \delta_{j}^{m}\right) .
$$

Further, $n^{\mu}$ is the future-pointing unit normal vector to the hypersurface $\Sigma_{t}$, which is written in terms of the ADM variables as

$$
n^{0}=\sqrt{-g^{00}}=\frac{1}{N}, \quad n^{i}=-\frac{g^{0 i}}{\sqrt{-g^{00}}}=-\frac{N^{i}}{N} .
$$

Inserting these results to the action and performing integration by parts we obtain the action in the form

$$
\begin{aligned}
S\left[N, N^{i}, h_{i j}, \Phi, \lambda, \phi\right]= & \frac{1}{2} \int d t d^{3} \mathbf{x} \sqrt{h} N\left[K_{i j} \mathcal{G}^{i j k l} K_{k l} \Phi^{2}+R \Phi^{2}-4 K \Phi \nabla_{n} \Phi\right. \\
& -\frac{2}{\sqrt{h}} \partial_{i}\left(\sqrt{h} h^{i j} \partial_{j} \Phi^{2}\right)-6\left(\nabla_{n} \Phi\right)^{2}+6 h^{i j} \partial_{i} \Phi \partial_{j} \Phi \\
& \left.-\lambda \Phi^{2}+\lambda\left(\nabla_{n} \phi\right)^{2}-\lambda h^{i j} \partial_{i} \phi \partial_{j} \phi\right],
\end{aligned}
$$

where

$$
\nabla_{n} \Phi=\frac{1}{N}\left(\partial_{t} \Phi-N^{i} \partial_{i} \Phi\right),
$$

and where we ignored boundary terms. Now we can easily derive the momenta conjugate to $h_{i j}, \Phi, \lambda$ and $\phi$ from the action (2.14) as

$$
\begin{aligned}
\pi^{i j} & =\frac{1}{2} \sqrt{g} \mathcal{G}^{i j k l} K_{k l} \Phi^{2}-\sqrt{h} h^{i j} \nabla_{n} \Phi \Phi, \\
p_{\Phi} & =-2 K \Phi \sqrt{h}-6 \sqrt{h} \nabla_{n} \Phi, \\
p_{\lambda} & \approx 0, \quad p_{\phi}=\sqrt{h} \lambda \nabla_{n} \phi .
\end{aligned}
$$

Using these relations we obtain the following primary constraint

$$
\mathcal{D}=p_{\Phi} \Phi-2 \pi^{i j} h_{i j} \approx 0
$$

and the Hamiltonian in the form

$$
\begin{aligned}
H= & \int d^{3} \mathbf{x}\left(N \mathcal{H}_{T}+N^{i} \mathcal{H}_{i}+v_{\mathcal{D}} \mathcal{D}+v_{N} \pi_{N}+v^{i} \pi_{i}+v_{\lambda} p_{\lambda}\right), \\
\mathcal{H}_{T}= & \frac{2}{\sqrt{h} \Phi^{2}} \pi^{i j} \mathcal{G}_{i j k l} \pi^{k l}-\frac{1}{2} \sqrt{h} R \Phi^{2}+\frac{1}{2 \sqrt{h} \lambda} p_{\phi}^{2}+\partial_{i}\left(\sqrt{h} h^{i j} \partial_{j} \Phi^{2}\right) \\
& -3 \sqrt{h} h^{i j} \partial_{i} \Phi \partial_{j} \Phi+\frac{1}{2} \sqrt{h} \lambda\left(\Phi^{2}+h^{i j} \partial_{i} \phi \partial_{j} \phi\right), \\
\mathcal{H}_{i}= & p_{\Phi} \partial_{i} \Phi+p_{\phi} \partial_{i} \phi-2 h_{i j} D_{k} \pi^{j k} .
\end{aligned}
$$


Note that we have ignored the boundary contribution of the Hamiltonian, since we are interested in the behavior of the local degrees of freedom, rather than in the total gravitational energy. Therefore the Hamiltonian (2.18) is a sum of constraints that vanishes for any physical configuration on the constraint surface. In addition to the constraint terms, a complete Hamiltonian contains a surface term on the boundary of space, what defines the total energy of the system. The total energy is conserved in time, and according to the positive energy theorem of general relativity $[16,17]$ the total energy is positive, except for flat Minkowski spacetime, which has zero energy. ${ }^{2}$ Our analysis concerns the structure of the Hamiltonian density and the properties of the local degrees of freedom, and their constraints.

Now we proceed to the analysis of the preservation of the primary constraints $\pi_{N} \approx$ $0, \pi_{i} \approx 0, \mathcal{D} \approx 0, p_{\lambda} \approx 0$. As usual the requirement of the preservation of the constraints $\pi_{N}, \pi_{i}$ implies the secondary constraints

$$
\mathcal{H}_{T} \approx 0, \quad \mathcal{H}_{i} \approx 0 .
$$

For further analysis we introduce the smeared form of these constraints

$$
\mathbf{T}_{T}(N)=\int d^{3} \mathbf{x} N \mathcal{H}_{T}, \quad \mathbf{T}_{S}\left(N^{i}\right)=\int d^{3} \mathbf{x}\left(N^{i} \mathcal{H}_{i}+p_{\lambda} \partial_{i} \lambda\right) .
$$

On the other hand the requirement of the preservation of the constraint $p_{\lambda}$ implies

$$
\frac{1}{N} \partial_{t} p_{\lambda}=\frac{1}{N}\left\{p_{\lambda}, H\right\}=\frac{1}{2 \sqrt{h} \lambda^{2}} p_{\phi}^{2}-\frac{1}{2} \sqrt{h}\left(\Phi^{2}+h^{i j} \partial_{i} \phi \partial_{j} \phi\right) \equiv \mathcal{C}_{\lambda} \approx 0 .
$$

Let us now proceed to the requirement of the preservation of the constraint $\mathcal{D}$. However, it is convenient to consider the following linear combination of $\mathcal{D}$ with the constraint $p_{\lambda} \approx 0$ in the form

$$
\tilde{\mathcal{D}}=\mathcal{D}+2 p_{\lambda} \lambda=p_{\Phi} \Phi-2 \pi^{i j} h_{i j}+2 p_{\lambda} \lambda,
$$

which has the following non-zero Poisson brackets:

$$
\begin{gathered}
\left\{\tilde{\mathcal{D}}(\mathbf{x}), h_{i j}(\mathbf{y})\right\}=2 h_{i j}(\mathbf{x}) \delta(\mathbf{x}-\mathbf{y}), \\
\left\{\tilde{\mathcal{D}}(\mathbf{x}), \pi^{i j}(\mathbf{y})\right\}=-2 \pi^{i j}(\mathbf{x}) \delta(\mathbf{x}-\mathbf{y}), \\
\{\tilde{\mathcal{D}}(\mathbf{x}), \Phi(\mathbf{y})\}=-\Phi(\mathbf{x}) \delta(\mathbf{x}-\mathbf{y}), \\
\left\{\tilde{\mathcal{D}}(\mathbf{x}), p_{\Phi}(\mathbf{y})\right\}=p_{\Phi}(\mathbf{x}) \delta(\mathbf{x}-\mathbf{y}), \\
\{\tilde{\mathcal{D}}(\mathbf{x}), \lambda(\mathbf{y})\}=-2 \lambda(\mathbf{x}) \delta(\mathbf{x}-\mathbf{y}), \\
\left\{\tilde{\mathcal{D}}(\mathbf{x}), p_{\lambda}(\mathbf{y})\right\}=2 p_{\lambda}(\mathbf{x}) \delta(\mathbf{x}-\mathbf{y}) .
\end{gathered}
$$

\footnotetext{
${ }^{2}$ The nonminimally coupled field $\Phi$ is not dynamical, since it is a gauge degree of freedom associated with the conformal symmetry. The total gravitational energy is independent of the chosen gauge for the conformal symmetry. When we fix the gauge of the conformal symmetry in section 2.1 , we obtain a minimally coupled scalar field theory, which is known to describe dust (see sections 2.2 and 3). We require that the energy density of the scalar field is positive on the initial Cauchy surface, say at time $t=0$, since only those initial configurations are physically meaningful. Then the energy conditions of the positive energy theorem of general relativity are satisfied at the inital time $t=0$ and the total gravitational energy is positive. Since the total energy is conserved, it remains positive. We will later argue that the system can become unstable when the energy density of the scalar field becomes negative under time evolution. In that case, the gravitational field should compensate for the contribution of the scalar field so that the total energy remains conserved.
} 
Then we obtain

$$
\left\{\tilde{\mathcal{D}}, \mathbf{T}_{T}(N)\right\}=-N \mathcal{H}_{T}
$$

using

$$
\delta R=D^{i} D^{j} \delta h_{i j}-h^{i j} D^{k} D_{k} \delta h_{i j}-R^{i j} \delta h_{i j} .
$$

In the same way we find

$$
\left\{\tilde{\mathcal{D}}, \mathbf{T}_{S}\left(N^{i}\right)\right\}=\partial_{i}\left(N^{i} \tilde{\mathcal{D}}\right)
$$

Collecting all these results together we find

$$
\partial_{t} \tilde{\mathcal{D}}=\left\{\tilde{\mathcal{D}}, H_{T}\right\}=-N \mathcal{H}_{T}+\partial_{i}\left(N^{i} \tilde{\mathcal{D}}\right)+2 v_{\lambda} p_{\lambda} \approx 0,
$$

so that $\tilde{\mathcal{D}}$ is preserved without imposing any additional constraint.

Finally we determine Poisson brackets between $\mathcal{H}_{T}$ and $\mathcal{H}_{i}$. We use their smeared form and we find

$$
\begin{aligned}
\left\{\mathbf{T}_{T}(N), \mathbf{T}_{T}(M)\right\}= & \mathbf{T}_{S}\left(\left(N \partial_{i} M-M \partial_{i} N\right) h^{i j}\right) \\
& -\int d^{3} \mathbf{x}\left(\partial_{i} M N-N \partial_{i} M\right) h^{i j} \frac{\partial_{j} \Phi}{\Phi} \mathcal{D}
\end{aligned}
$$

and we see that given expression vanishes on the constraint surface $\mathcal{H}_{i} \approx 0, \mathcal{D} \approx 0$. Further we have

$$
\left\{\mathbf{T}_{S}\left(N^{i}\right), \mathbf{T}_{S}\left(M^{i}\right)\right\}=\mathbf{T}_{S}\left(\left(N^{i} \partial_{i} M^{j}-M^{i} \partial_{i} N^{j}\right)\right)
$$

and finally

$$
\left\{\mathbf{T}_{S}\left(N^{i}\right), \mathbf{T}_{T}(M)\right\}=\mathbf{T}_{T}\left(N^{i} \partial_{i} M\right) .
$$

Hence the Hamiltonian and momentum constraints (2.19) are preserved under time evolution.

The number of physical degrees of freedom is obtained via Dirac's formula: (number of canonical variables) $/ 2$ - (number of first class constraints) - (number of second class constraints) $/ 2$. Compared to general relativity, we have six extra canonical variables $\left(\phi, p_{\phi}, \Phi, p_{\Phi}, \lambda, p_{\lambda}\right)$, one extra first class constraint $\tilde{\mathcal{D}} \approx 0$, and two extra second class constraints $p_{\lambda} \approx 0, \mathcal{C}_{\lambda} \approx 0$. Thus, in addition to the two gravitational degrees of freedom of general relativity, there exist one extra physical degree of freedom.

Now we see that $p_{\lambda} \approx 0$ and $\mathcal{C}_{\lambda} \approx 0$ are the second class constraints that can be set to vanish strongly. We solve the constraint $\mathcal{C}_{\lambda}=0$ with respect to $\lambda$ as

$$
\lambda= \pm \frac{p_{\phi}}{\sqrt{h} \sqrt{\Phi^{2}+h^{i j} \partial_{i} \phi \partial_{j} \phi}} .
$$

Inserting each of these two solutions into the Hamiltonian constraint defined in (2.18), we find that it is equal to

$$
\begin{aligned}
\mathcal{H}_{T}= & \frac{2}{\sqrt{h} \Phi^{2}} \pi^{i j} \mathcal{G}_{i j k l} \pi^{k l}-\frac{1}{2} \sqrt{h} R \Phi^{2}+\partial_{i}\left(\sqrt{h} h^{i j} \partial_{j} \Phi^{2}\right) \\
& -3 \sqrt{h} h^{i j} \partial_{i} \Phi \partial_{j} \Phi \pm p_{\phi} \sqrt{\Phi^{2}+h^{i j} \partial_{i} \phi \partial_{j} \phi}
\end{aligned}
$$


The pair of canonical variables $\lambda, p_{\lambda}$ has been eliminated from the formalism. Then we should determine the Dirac bracket between the remaining phase space variables $\pi^{i j}, h_{i j}$, $\phi, p_{\phi}$ and $\Phi, p_{\Phi}$. Recall that by definition the Dirac bracket between two phase space functions has the form

$$
\{A, B\}_{D}=\{A, B\}-\sum_{I, J}\left\{A, \Psi_{I}\right\}\left(\Omega^{-1}\right)^{I J}\left\{\Psi_{J}, B\right\},
$$

where $\Psi^{I}, I=1,2$ are the second class constraints $p_{\lambda} \approx 0, \mathcal{C}_{\lambda} \approx 0$ and where $\Omega_{I J}$ is the matrix of the Poisson brackets

$$
\begin{aligned}
\Omega_{p_{\lambda}(\mathbf{x}), \mathcal{C}_{\lambda}(\mathbf{y})}= & \left\{p_{\lambda}(\mathbf{x}), \mathcal{C}_{\lambda}(\mathbf{y})\right\}=\frac{1}{\sqrt{h} \lambda^{3}} p_{\phi}^{2}(\mathbf{x}) \delta(\mathbf{x}-\mathbf{y}), \\
\Omega_{\mathcal{C}_{\lambda}(\mathbf{x}), \mathcal{C}_{\lambda}(\mathbf{y})}= & \frac{1}{\sqrt{h} \lambda^{2}(\mathbf{x})} p_{\phi}(\mathbf{x}) \sqrt{h} h^{i j}(\mathbf{y}) \partial_{y^{j}} \delta(\mathbf{x}-\mathbf{y}) \partial_{y^{i}} \phi(\mathbf{y}) \\
& -\frac{1}{\sqrt{h} \lambda^{2}(\mathbf{y})} p_{\phi}(\mathbf{y}) \sqrt{h} h^{i j}(\mathbf{x}) \partial_{x^{j}} \delta(\mathbf{x}-\mathbf{y}) \partial_{x^{i}} \phi(\mathbf{x}),
\end{aligned}
$$

so that the matrix $\Omega$ has the following schematic form

$$
\Omega=\left(\begin{array}{cc}
0 & A \\
-A & B
\end{array}\right)
$$

and its inverse has the form

$$
\Omega^{-1}=\left(\begin{array}{cc}
A^{-1} B A^{-1} & -A^{-1} \\
A^{-1} & 0
\end{array}\right) .
$$

Now we find that the Dirac brackets coincides with the Poisson brackets due to the fact that $\left\{h_{i j}, p_{\lambda}\right\}=\left\{\pi^{i j}, p_{\lambda}\right\}=\left\{p_{\phi}, p_{\lambda}\right\}=\left\{\phi, p_{\lambda}\right\}=0$.

Note that the scalar part of the Hamiltonian has a similar structure as in the case of Lagrange multiplier modified gravities, see [9]. The reason for this becomes evident in section 3, where we find that by gauge fixing the conformal symmetry we can write the theory as a certain type of Lagrange multiplier modified gravity.

Let us now return to the Hamiltonian with the second class constraints solved. From (2.32) we see that the Hamiltonian constraint depends linearly on the momentum $p_{\phi}$. In some cases such a linear dependence implies that the Hamiltonian density is not bounded from below, which is a classical sign of instability. This is especially the case in many higher derivative field theories, which are notorious for their Ostrogradskian instability (for discussion and example, see $[18,19]$ ). On the other hand, in certain cases a linear dependence on a canonical momentum does not imply instability. As a very simple example we mention the Hamiltonian of a harmonic oscillator written in terms of action-angle variables, $H=\omega P$, where $\omega$ is the angular frequency and $P$ is the momentum conjugate to the action-angle coordinate (see, e.g., [20]). Since a momentum that is conjugate to an action-angle coordinate is a constant of motion, the previous Hamiltonian is a constant and hence trivially bounded from below. Thus, in order to determine whether the present 
theory of mimetic dark matter [1] involves an instability, we have to study the dynamics of the variables $\phi, p_{\phi}$ carefully. In the present case, the stability of the system depends on whether the momentum $\pm p_{\phi}$ in the two alternative Hamiltonians given by (2.32) can evolve to the negative side of the phase space, $\pm p_{\phi}<0$, and eventually to negative infinity, $\pm p_{\phi} \rightarrow-\infty$. If that happens, the system is unstable. Note that when the kinetic $p_{\phi}$-term becomes negative, the metric/tensor part of the Hamiltonian constraint $\mathcal{H}_{T}$ has to compensate for it by increasing its local value, since the Hamiltonian constraint must remain zero for every physical configuration. Consequently, the system could be driven to an increasingly excited state, one kinetic term towards negative infinity and another term towards positive infinity, and hence a stable vacuum could not exist.

We will later show that the two alternative Hamiltonians given by (2.32) actually describe the same physical system. Therefore it suffices to consider the dynamics for one of the cases, which we choose to be the one with the linear dependence on $+p_{\phi}$. Namely, we consider the Hamiltonian given by

$$
\begin{aligned}
\mathcal{H}_{T}= & \frac{2}{\sqrt{h} \Phi^{2}} \pi^{i j} \mathcal{G}_{i j k l} \pi^{k l}-\frac{1}{2} \sqrt{h} R \Phi^{2}+\partial_{i}\left(\sqrt{h} h^{i j} \partial_{j} \Phi^{2}\right) \\
& -3 \sqrt{h} h^{i j} \partial_{i} \Phi \partial_{j} \Phi+p_{\phi} \sqrt{\Phi^{2}+h^{i j} \partial_{i} \phi \partial_{j} \phi}
\end{aligned}
$$

Physically, the momentum $p_{\phi}$ is proportional to the energy density of the mimetic dust on the spatial hypersurface $\Sigma_{t}$. More precisely, $p_{\phi}$ is the rest mass density of the mimetic dust per coordinate volume element $d^{3} x$, as measured by the Eulerian observers with fourvelocity $n^{\mu}$. Since $p_{\phi}$ has the physical meaning of density of rest mass, we require that $p_{\phi}$ is initially nonnegative everywhere. That is the initial configuration of the system must satisfy $p_{\phi} \geq 0$ everywhere on the initial Cauchy surface, say $\Sigma_{0}$ at time $t=0$. The physical meaning of $\phi$ is that its gradient $\partial_{\mu} \phi$ is the direction of the rest mass current of the mimetic dust in spacetime. Then let us discuss the dynamics of the system and in particular the dynamics of the mimetic dust. We obtain the equation of motion for $\phi$ in the form

$$
\partial_{t} \phi=\{\phi, H\}=N \sqrt{\Phi^{2}+h^{i j} \partial_{i} \phi \partial_{j} \phi}+N^{i} \partial_{i} \phi
$$

The square of this equation gives the relation of $\Phi$ to $\phi$ as $\Phi^{2}=\left(\nabla_{n} \phi\right)^{2}-h^{i j} \partial_{i} \phi \partial_{j} \phi=$ $-g^{\mu \nu} \partial_{\mu} \phi \partial_{\nu} \phi$, which tells that the rest mass current of the mimetic dust is a timelike vector in spacetime. From the equation of motion (2.38) we obtain that the time evolution of $\phi$ does not depend on $p_{\phi}$, which is a rather strange equation of motion. This kind of systems where the evolution of a coordinate does not depend on canonical momenta have been studied in the past in the context of 't Hooft's deterministic quantum mechanics [21-24]. Presuming a gauge where $N=$ positive constant, $N^{i}=0$ and $\Phi=$ positive constant, we see from (2.38) that $\phi$ experiences monotonic and eternal growth under time evolution. The rate of growth has the minimal value of $\partial_{t} \phi=N \Phi$ and it is speed up by the presence of spatial nonhomogeneity in $\phi$ so that the norm of the spacetime gradient $\partial_{\mu} \phi$ remains constant. The spatial gradient of $\phi$ is the dynamically relevant quantity, while the local value of $\phi$ on $\Sigma_{t}$ is physically irrelevant. 
The equation of motion for the momentum $p_{\phi}$ has the form

$$
\partial_{t} p_{\phi}=\left\{p_{\phi}, H\right\}=\partial_{i}\left(\frac{N p_{\phi} h^{i j} \partial_{j} \phi}{\sqrt{\Phi^{2}+h^{i j} \partial_{i} \phi \partial_{j} \phi}}+N^{i} p_{\phi}\right) .
$$

Physically this is the continuity equation for the rest mass current of the mimetic dust, which ensures that the total rest mass on the spatial hypersurface $\Sigma_{t}$ is conserved under time evolution from one hypersurface to the next. First we would like to find the configuration of this system which could be interpreted as the ground state in the sense that the time derivative of $p_{\phi}$ is equal to zero. We can rewrite the equation of motion (2.39) as

$$
\partial_{t} p_{\phi}=p_{\phi} \partial_{i}\left(\frac{N h^{i j} \partial_{j} \phi}{\sqrt{\Phi^{2}+h^{i j} \partial_{i} \phi \partial_{j} \phi}}+N^{i}\right)+\partial_{i} p_{\phi}\left(\frac{N h^{i j} \partial_{j} \phi}{\sqrt{\Phi^{2}+h^{i j} \partial_{i} \phi \partial_{j} \phi}}+N^{i}\right) .
$$

From (2.40) we see that there exists a ground state where $p_{\phi}=0$. If there exists a region of space where $p_{\phi}=0$, then inside that region $p_{\phi}$ remains zero, since $p_{\phi}=0$ and $\partial_{i} p_{\phi}=0$ imply that $\partial_{t} p_{\phi}=0$. Then let us consider an initial configuration where $p_{\phi}>0$ (inside some region or everywhere in space), which corresponds to the presence of mimetic dust. Now the crucial question is whether $p_{\phi}$ can evolve to the negative side of the phase space $p_{\phi}<0$. If that can happen, the system is unstable. Indeed, since negative $p_{\phi}$ means dust with negative rest mass, an infinite amount of radiation, matter or dust could be created without violating the conservation of total energy. The question has two steps: can $p_{\phi}$ evolve to zero, and if it does, can it become negative? Assuming the aforementioned gauge, we obtain the equation of motion (2.40) as

$$
\partial_{t} p_{\phi}=N p_{\phi} \partial_{i}\left(\frac{h^{i j} \partial_{j} \phi}{\sqrt{\Phi^{2}+h^{i j} \partial_{i} \phi \partial_{j} \phi}}\right)+\frac{N h^{i j} \partial_{i} p_{\phi} \partial_{j} \phi}{\sqrt{\Phi^{2}+h^{i j} \partial_{i} \phi \partial_{j} \phi}} .
$$

Since the evolution of $\phi$ (and of its gradient $\partial_{i} \phi$ ) with time is independent of $p_{\phi}$, we can setup the configuration for $\phi$ freely when we consider the dynamics of $p_{\phi}$. Consider a (small) region of space where the metric $h_{i j}$ and the gradient $\partial_{i} \phi$ are nearly constant. Then the equation of motion (2.41) is dominated by its second term, while the first term is very small in comparison. Furthermore, we consider that the gradient $\partial_{i} \phi$ is contradirectional compared to the gradient $\partial_{i} p_{\phi}$ so that $h^{i j} \partial_{i} p_{\phi} \partial_{j} \phi<0$. For example, let us consider that the given point is a local minimum of $p_{\phi}$, so that $\partial_{i} p_{\phi}$ is pointing away from the given point. Thus, regardless of how close $p_{\phi}$ is to zero, it can evolve towards zero, since $\partial_{t} p_{\phi}$ can be negative. There appears to be nothing that could stop $p_{\phi}$ from evolving to zero, since the time evolution of $p_{\phi}$ does not necessarily change the direction of the gradient $\partial_{i} p_{\phi}$ so that $h^{i j} \partial_{i} p_{\phi} \partial_{j} \phi$ would become nonnegative. However, proving this decisively would require an exact solution that crosses the point $p_{\phi}=0$. Since such a solution must be nonhomogeneous and nonisotropic, it is very hard to achieve. Alternatively, one could try to show that on some background the perturbation of $p_{\phi}$ can grow to negative infinity. Let us then consider what happens assuming that $p_{\phi}$ has evolved to zero. Now the equation of motion (2.41) reads as

$$
\partial_{t} p_{\phi}=\frac{N h^{i j} \partial_{i} p_{\phi} \partial_{j} \phi}{\sqrt{\Phi^{2}+h^{i j} \partial_{i} \phi \partial_{j} \phi}}
$$


When the directions of the gradients of $p_{\phi}$ and $\phi$ are such that $h^{i j} \partial_{i} p_{\phi} \partial_{j} \phi<0$, we obtain that $\partial_{t} p_{\phi}<0$ and consequently $p_{\phi}$ becomes negative. Thus, our arguments indicate that under certain circumstances, the energy density of the mimetic dust can become negative, and consequently the system can become unstable.

Let us consider the Hamiltonian constraint (2.32) with the negative sign in front of $p_{\phi}$. Now $-p_{\phi}$ has the physical meaning as the rest mass density of the mimetic dust. Hence $p_{\phi}$ must be negative initially. The equations of motion are obtained as

$$
\partial_{t} \phi=\{\phi, H\}=-N \sqrt{\Phi^{2}+h^{i j} \partial_{i} \phi \partial_{j} \phi}+N^{i} \partial_{i} \phi
$$

and

$$
\partial_{t} p_{\phi}=\left\{p_{\phi}, H\right\}=\partial_{i}\left(-\frac{N p_{\phi} h^{i j} \partial_{j} \phi}{\sqrt{\Phi^{2}+h^{i j} \partial_{i} \phi \partial_{j} \phi}}+N^{i} p_{\phi}\right) .
$$

We can see that this system is simply the mirror image of the system considered above obtained via the transformation $\left(\phi, p_{\phi}\right) \rightarrow\left(-\phi,-p_{\phi}\right)$.

We briefly consider the inclusion of a potential term for the scalar field $\phi$. It would be included into the Hamiltonian constraint $\mathcal{H}_{T}$ by adding $\sqrt{h} \Phi^{4} V(\phi)$, where the potential $V(\phi)$ is a local function of $\phi$. Then it would contribute an extra term into the right-hand side of the equation of motion (2.39) as $-N \sqrt{h} \Phi^{4} \frac{d V(\phi)}{d \phi}$. When this term is positive, it raises the bar for the appearance of the instability, but this does not change the conclusion. The system can still become unstable for the given kind of initial configurations.

Despite the potential problem of instability discussed above the original theory of mimetic dark matter could be useful for astrophysical and cosmological modeling, provided that one considers only those initial configurations that do not cross the point $p_{\phi}=0$ under time evolution. Those are the cases that describe physical dust.

\subsection{Gauge fixing of scale symmetry}

Returning to our Hamiltonian formulation we fix the dilatation symmetry by introducing the gauge fixing function

$$
\mathcal{C}_{\text {scale }} \equiv \Phi-1=0
$$

Clearly we have

$$
\left\{\mathcal{C}_{\text {scale }}(\mathbf{x}), \mathcal{D}(\mathbf{y})\right\}=\delta(\mathbf{x}-\mathbf{y})
$$

and hence they are the second class constraints. We can also explicitly solve $\mathcal{D}$ for $p_{\Phi}$ and we obtain

$$
p_{\Phi}=2 \pi^{i j} h_{i j} .
$$

Then the Hamiltonian constraint has the form

$$
\mathcal{H}_{T}=\mathcal{H}_{T}^{\mathrm{GR}}+\mathcal{H}_{T}^{\phi} \approx 0,
$$

where $\mathcal{H}_{T}^{\mathrm{GR}}$ is the standard contribution of general relativity,

$$
\mathcal{H}_{T}^{\mathrm{GR}}=\frac{2}{\sqrt{h}} \pi^{i j} \mathcal{G}_{i j k l} \pi^{k l}-\frac{1}{2} \sqrt{h} R,
$$


and the contribution of the scalar field is given as

$$
\mathcal{H}_{T}^{\phi}=\frac{1}{2 \sqrt{h} \lambda} p_{\phi}^{2}+\frac{1}{2} \sqrt{h} \lambda\left(1+h^{i j} \partial_{i} \phi \partial_{j} \phi\right) .
$$

The momentum constraint is given as

$$
\mathcal{H}_{i}=p_{\phi} \partial_{i} \phi-2 h_{i j} D_{k} \pi^{j k} \approx 0 .
$$

Solving the second class constraints $p_{\lambda} \approx 0$ and

$$
\mathcal{C}_{\lambda}=\frac{1}{2 \sqrt{h} \lambda^{2}} p_{\phi}^{2}+\frac{1}{2} \sqrt{h}\left(1+h^{i j} \partial_{i} \phi \partial_{j} \phi\right) \approx 0,
$$

we again find the contribution of the scalar $\phi$ in the form

$$
\mathcal{H}_{T}^{\phi}=p_{\phi} \sqrt{1+h^{i j} \partial_{i} \phi \partial_{j} \phi},
$$

where we chose the solution (2.31) with the positive sign. Now only the first class constraints which are associated with the diffeomorphism invariance remain, namely $\pi_{N} \approx 0$, $\pi_{i} \approx 0, \mathcal{H}_{T} \approx 0, \mathcal{H}_{i} \approx 0$. There exists an extra scalar degree of freedom that is associated with the variables $\phi, p_{\phi}$. It couples to the metric via the square root factor in (2.53). The term that is linear in the momentum $p_{\phi}$ persists and it has the same form and dynamics which were discussed above.

\subsection{Dust structure of the Hamiltonian}

If we assume that $\lambda>0$ everywhere in spacetime, and consequently that $p_{\phi}>0$, we obtain the contribution of the scalar field (2.53) in the Hamiltonian (2.48) as

$$
\mathcal{H}_{T}^{\phi}=\sqrt{p_{\phi}^{2}+h^{i j} \mathcal{H}_{i}^{\phi} \mathcal{H}_{j}^{\phi}}
$$

where $\mathcal{H}_{i}^{\phi}$ denotes the contribution of $\phi$ to the momentum constraint in (2.18),

$$
\mathcal{H}_{i}^{\phi}=p_{\phi} \partial_{i} \phi
$$

The same Hamiltonian for dust was obtained in [25] using eight scalar fields on spacetime to describe the full dynamics of dust. Once the conformal gauge is fixed (see section 2.1) the mimetic theory contains only two extra scalars, namely the field $\phi$ and the solvable Lagrange multiplier $\lambda$. In [25], these two scalars are denoted by $T$ and $M$, respectively. $M$ was assumed to be positive since it represents the rest mass density of the dust. Compared to [25] the present mimetic model lacks both the dust frame fields $Z^{k}(k=1,2,3)$ and the spatial components $W_{k}$ of the four-velocity of dust in the dust frame. Thus we conclude that the mimetic theory [1] with the assumption $\lambda>0$, and with the conformal symmetry gauge fixed, is a reduced version of the model of dust that was studied in [25].

The problem with imposing the condition $\lambda>0$ is that it appears to be inconsistent with the equations of motion in some cases. When $\lambda$ is solved (2.31), the requirement $\lambda>0$ becomes the requirement $p_{\phi}>0$ (or $p_{\phi}<0$ for the second solution). Since $p_{\phi}$ is 
the density of the mimetic dust, we require that $p_{\phi}>0$ on the initial Cauchy surface. The arguments presented above indicate that for some initial configurations, $p_{\phi}$ could evolve to zero and further to $p_{\phi}<0$. Hence the requirement $\lambda>0$ is not always consistent with the dynamics. Actually, the requirement $\lambda>0$ appears to be equivalent to picking up the initial configurations that do no cross the surface $p_{\phi}=0$ under time evolution.

\section{Mimetric theory in Einstein frame}

In this section, we present the formulation of the mimetric theory in the Einstein frame. We can rewrite the action (2.5) in the Einstein frame by gauge fixing the dilatation symmetry. When we perform the conformal transformation $g_{\mu \nu} \rightarrow \Omega^{2} g_{\mu \nu}$, using

$$
R\left(g_{\mu \nu}\right) \rightarrow \frac{1}{\Omega^{2}}\left(R\left(g_{\mu \nu}\right)-6 \frac{g^{\mu \nu} \nabla_{\mu} \nabla_{\nu} \Omega}{\Omega}\right)
$$

and with the scale fixed as $\Omega=\Phi^{-1}$, the action (2.5) takes the following form

$$
S\left[g_{\mu \nu}, \lambda, \phi\right]=\frac{1}{2} \int d^{4} x \sqrt{-g}\left[R\left(g_{\mu \nu}\right)-\lambda\left(1+g^{\mu \nu} \nabla_{\mu} \phi \nabla_{\nu} \phi\right)\right],
$$

where $\lambda$ is the Lagrange multiplier that ensures the spacetime gradient $\nabla_{\mu} \phi$ is unit and timelike. The same action can be obtained by simply setting the conformal gauge in (2.5) as $\Phi=1$. Thus the conformal invariance is necessarily lost in the Einstein frame. This reduced form of the mimetic theory was first obtained in [26] but in another way. Now we see that in the Einstein frame the mimetic theory reduces to Lagrange multiplier modified gravity [6] without any extra terms present in the Lagrangian except the constraint multiplied by $\lambda$. The Hamiltonian analysis of Lagrange multiplier modified theory of gravity was performed in [9] with additional kinetic and potential terms included. The Hamiltonian for the action (3.2) is the same one that was obtained in section 2.1 via gauge fixing the conformal symmetry of the mimetic theory with (2.45). In other words, the formulation in Einstein frame represents one conformal gauge of the mimetic theory. As usual, there exist alternative gauges.

\section{Vector field model of mimetic dark matter}

The vector field model of mimetic dark matter theory was suggested in [2]. It is based on the presumption that the physical metric has the form

$$
g_{\mu \nu}^{\text {phys }}=-\left(g^{\alpha \beta} u_{\alpha} u_{\beta}\right) g_{\mu \nu} \equiv \Phi^{2} g_{\mu \nu} .
$$

The action that now contains the Maxwell kinetic term has the form

$$
S\left[g_{\mu \nu}^{\text {phys }}, u_{\mu}\right]=\int d^{4} x \sqrt{-g^{\text {phys }}}\left[\frac{1}{2} R\left(g_{\mu \nu}^{\text {phys }}\right)-\frac{\mu^{2}}{4} g_{\text {phys }}^{\mu \alpha} g_{\text {phys }}^{\nu \beta} F_{\mu \nu} F_{\alpha \beta}\right],
$$

where

$$
g_{\mathrm{phys}}^{\mu \nu}=\Phi^{-2} g^{\mu \nu}
$$


and

$$
F_{\mu \nu}=\partial_{\mu} u_{\nu}-\partial_{\nu} u_{\mu},
$$

and where $\mu^{2}$ is the parameter having the dimension of mass squared.

Our goal is to perform the Hamiltonian analysis of given action. We follow the analysis performed in the case of scalar action so that we obtain ${ }^{3}$

$$
\begin{aligned}
S\left[g_{\mu \nu}, \Phi, \lambda, u_{\mu}\right]= & \int d^{4} x \sqrt{-g}\left[\frac{1}{2} \Phi^{2} R\left(g_{\mu \nu}\right)+3 g^{\mu \nu} \nabla_{\mu} \Phi \nabla_{\nu} \Phi\right. \\
& \left.+\frac{1}{2} \lambda\left(\Phi^{2}+g^{\mu \nu} u_{\mu} u_{\nu}\right)-\frac{\mu^{2}}{4} g^{\mu \alpha} g^{\nu \beta} F_{\mu \nu} F_{\alpha \beta}\right] .
\end{aligned}
$$

Now we are ready to proceed to the Hamiltonian formalism of given action. The gravitational part is the same as before. On the other hand in case of the vector part we closely follow [12] and find that the action for the vector field has the form

$$
\begin{aligned}
S_{u}= & -\frac{\mu^{2}}{4} \int d^{4} x \sqrt{-g} g^{\mu \alpha} g^{\nu \beta} F_{\mu \nu} F_{\alpha \beta} \\
= & -\frac{\mu^{2}}{4} \int d t d^{3} \mathbf{x} \sqrt{h} N\left[h^{i k} h^{j l}\left(D_{i} u_{j}-D_{j} u_{i}\right)\left(D_{k} u_{l}-D_{l} u_{k}\right)\right. \\
& \left.-2 h^{i j}\left(\mathcal{L}_{n} u_{i}-a_{i} u_{\mathbf{n}}-D_{i} u_{\mathbf{n}}\right)\left(\mathcal{L}_{n} u_{j}-a_{j} u_{\mathbf{n}}-D_{j} u_{\mathbf{n}}\right)\right],
\end{aligned}
$$

where

$$
\mathcal{L}_{n} u_{i}=\frac{1}{N}\left(\partial_{t} u_{i}-\mathcal{L}_{\vec{N}} u_{i}\right)=\frac{1}{N}\left(\partial_{t} u_{i}-N^{k} \partial_{k} u_{i}-\partial_{i} N^{k} u_{k}\right), \quad a_{i}=\frac{D_{i} N}{N} .
$$

Using this form of the action we can easily proceed to the Hamiltonian formulation. First of all we have following conjugate momenta $p^{i}, p_{\mathbf{n}}$ to $u_{i}$ and $u_{\mathbf{n}}$, respectively

$$
p^{i}=\frac{\delta L}{\delta \partial_{t} u_{i}}=\mu^{2} \sqrt{h} h^{i j}\left(\mathcal{L}_{n} u_{j}-a_{j} u_{\mathbf{n}}-D_{j} u_{\mathbf{n}}\right), \quad p_{\mathbf{n}} \approx 0
$$

Then it is easy to find the Hamiltonian for given system

$$
\begin{aligned}
H= & \int d^{3} \mathbf{x}\left(N \mathcal{H}_{T}+N^{i} \mathcal{H}_{i}+v_{\mathcal{D}} \mathcal{D}+v_{N} \pi_{N}+v^{i} \pi_{i}+v_{\lambda} p_{\lambda}+v_{\mathbf{n}} p_{\mathbf{n}}\right), \\
\mathcal{H}_{T}= & \frac{2}{\sqrt{h} \Phi^{2}} \pi^{i j} \mathcal{G}_{i j k l} \pi^{k l}-\frac{1}{2} \sqrt{h} R \Phi^{2}+\partial_{i}\left[\sqrt{h} h^{i j} \partial_{j} \Phi^{2}\right] \\
& -3 \sqrt{h} h^{i j} \partial_{i} \Phi \partial_{j} \Phi-\frac{1}{2} \sqrt{h} \lambda\left(\Phi^{2}+u_{i} h^{i j} u_{j}-u_{\mathbf{n}}^{2}\right) \\
& +\frac{1}{2 \mu^{2} \sqrt{h}} p^{i} h_{i j} p^{j}+\frac{\mu^{2}}{4} \sqrt{h} h^{i k} h^{j l}\left(D_{i} u_{j}-D_{j} u_{i}\right)\left(D_{k} u_{l}-D_{l} u_{k}\right)-u_{\mathbf{n}} D_{i} p^{i}, \\
\mathcal{H}_{i}= & p_{\Phi} \partial_{i} \Phi-2 h_{i k} D_{j} \pi^{k j}+\partial_{i} u_{j} p^{j}-\partial_{j}\left(u_{i} p^{j}\right) .
\end{aligned}
$$

\footnotetext{
${ }^{3}$ In principle there is no compelling reason why to introduce $\Phi$ as an independent variable. However, the presence of the term $g^{\mu \nu} \nabla_{\mu} \Phi \nabla_{\nu} \Phi$ in the action would imply the following expression $\Phi^{-2} g^{\mu \nu}\left(\nabla_{\mu} u_{\rho} g^{\rho \sigma} u_{\sigma}\right)\left(\nabla_{\nu} u_{\gamma} g^{\gamma \delta} u_{\delta}\right)$ that would lead to very complicated expression when we implemented $3+1$ decomposition of the metric and the vector field $u_{\mu}$. For that reason we still treat $\Phi$ as an independent variable exactly as in the previous section.
} 
Note that we have

$$
\left\{\mathbf{T}_{S}\left(N^{i}\right), u_{j}(\mathbf{x})\right\}=-N^{k} \partial_{k} u_{i}-\partial_{i} N^{k} u_{k}
$$

Now we can proceed with the analysis in the same way as in previous section. We have the following set of the primary constraints ${ }^{4}$

$$
\begin{aligned}
\tilde{\mathcal{D}} & =p_{\Phi} \Phi-2 \pi^{i j} h_{i j}+2 p_{\lambda} \lambda+p_{\mathbf{n}} u_{\mathbf{n}} \approx 0, \\
p_{\lambda} & \approx 0, \quad \pi_{N} \approx 0, \quad \pi_{i} \approx 0, \quad p_{\mathbf{n}} \approx 0 .
\end{aligned}
$$

The preservation of the primary constraints $p_{N}, p^{i}$ during the time evolution of the system implies following secondary constraints

$$
\mathcal{H}_{T} \approx 0, \quad \mathcal{H}_{i} \approx 0
$$

while the constraint $\tilde{\mathcal{D}}$ is preserved and it is the first class constraint that is the generator of the scaling transformation. On the other hand the requirement of the preservation of the constraint $p_{\lambda} \approx 0$ implies

$$
\frac{1}{N} \partial_{t} p_{\lambda}=\frac{1}{N}\left\{p_{\lambda}, H\right\}=\frac{1}{2} \sqrt{h}\left(\Phi^{2}+h^{i j} u_{i} u_{j}-u_{\mathbf{n}}^{2}\right) \equiv \frac{1}{2} \mathcal{C}_{\lambda} \approx 0 .
$$

Finally the requirement of the preservation of the constraint $p_{\mathbf{n}}$ gives

$$
\frac{1}{N} \partial_{t} p_{\mathbf{n}}=\frac{1}{N}\left\{p_{\mathbf{n}}, H\right\}=-\sqrt{h} \lambda u_{\mathbf{n}}+D_{i} p^{i} \equiv \mathcal{C}_{\mathbf{n}} \approx 0
$$

To proceed further note that we have following non-zero Poisson brackets

$$
\begin{aligned}
& \left\{p_{\lambda}(\mathbf{x}), \mathcal{C}_{\mathbf{n}}(\mathbf{y})\right\}=\sqrt{h} u_{\mathbf{n}}(\mathbf{x}) \delta(\mathbf{x}-\mathbf{y}) \\
& \left\{p_{\mathbf{n}}(\mathbf{x}), \mathcal{C}_{\mathbf{n}}(\mathbf{y})\right\}=\sqrt{h} \lambda(\mathbf{x}) \delta(\mathbf{x}-\mathbf{y}) \\
& \left\{p_{\mathbf{n}}(\mathbf{x}), \mathcal{C}_{\lambda}(\mathbf{y})\right\}=2 \sqrt{h} u_{\mathbf{n}}(\mathbf{x}) \delta(\mathbf{x}-\mathbf{y})
\end{aligned}
$$

which show that $p_{\lambda}, \mathcal{C}_{\mathbf{n}}, p_{\mathbf{n}}$ and $\mathcal{C}_{\lambda}$ are the second class constraints. Considering the Hamiltonian constraint, we find after some calculations

$$
\begin{aligned}
\left\{\mathbf{T}_{T}(N), \mathbf{T}_{T}(M)\right\}= & \mathbf{T}_{S}\left(\left(N \partial_{i} M-M \partial_{i} N\right) h^{i j}\right) \\
& -\int d^{3} \mathbf{x}\left(\partial_{i} M N-N \partial_{i} M\right) h^{i j} \frac{\partial_{j} \Phi}{\Phi} \mathcal{D} \\
& -\int d^{3} \mathbf{x}\left(\partial_{i} M N-M \partial_{i} M\right) h^{i j} u_{j} \mathcal{C}_{\mathbf{n}} .
\end{aligned}
$$

This again implies that $\mathcal{H}_{T}$ and $\mathcal{H}_{i}$ are the first class constraints which is the reflection of the diffeomorphism invariance of given theory.

\footnotetext{
${ }^{4}$ Note that it is not surprising that we include the expression $p_{\mathbf{n}} u_{\mathbf{n}}$ into the definition of the dilatation constraint, since it can be easily shown that $u_{\mathbf{n}}$ transforms non-trivially under (1.2). In fact, by definition we have $u_{\mathbf{n}}=n^{\mu} u_{\mu}=\frac{1}{N}\left(u_{0}-N^{i} u_{i}\right)$ and since $N, N^{i}$ transform under (1.2) as $N^{\prime}=\Omega N, N_{i}^{\prime}=\Omega^{2} N_{i}, N^{\prime i}=N^{i}$, we easily find that $u_{\mathbf{n}}^{\prime}=-\frac{1}{\Omega} u_{\mathbf{n}}$.
} 
Let us now return to the second class constraints in given model. We saw that $\mathcal{C}_{\mathbf{n}}$ and $\mathcal{C}_{\lambda}$ are the second class constraints that vanish strongly. From $\mathcal{C}_{\lambda}$ we can express $u_{\mathbf{n}}$ as

$$
u_{\mathbf{n}}=-\sqrt{\Phi^{2}+u_{i} h^{i j} u_{j}}
$$

where we chose $u_{\mathbf{n}}$ to be negative for a reason that will be clear below. The sign of $u_{\mathbf{n}}$ can be chosen since $u_{\mathbf{n}}$ is no longer an independent variable. Further, from $\mathcal{C}_{\mathbf{n}}$ we express $\lambda$ as

$$
\lambda=-\frac{D_{i} p^{i}}{\sqrt{h} \sqrt{\Phi^{2}+u_{i} h^{i j} u_{j}}} .
$$

Using these results we find the Hamiltonian constraint in the form

$$
\begin{aligned}
\mathcal{H}_{T}= & \frac{2}{\sqrt{h} \Phi^{2}} \pi^{i j} \mathcal{G}_{i j k l} \pi^{k l}-\frac{1}{2} \sqrt{h} R \Phi^{2}+\partial_{i}\left[\sqrt{h} h^{i j} \partial_{j} \Phi^{2}\right] \\
& -3 \sqrt{h} h^{i j} \partial_{i} \Phi \partial_{j} \Phi+\frac{1}{2 \mu^{2} \sqrt{h}} p^{i} h_{i j} p^{j}+\sqrt{\Phi^{2}+u_{i} h^{i j} u_{j}} D_{i} p^{i} \\
& +\frac{\mu^{2}}{4} \sqrt{h} h^{i k} h^{j l}\left(D_{i} u_{j}-D_{j} u_{i}\right)\left(D_{k} u_{l}-D_{l} u_{k}\right) .
\end{aligned}
$$

There exist five physical degrees of freedom, since we have sixteen pairs of canonical variables, nine first class constraints $\left(\mathcal{H}_{T}, \mathcal{H}_{i}, \pi_{N}, \pi_{i}, \mathcal{D}\right)$ and four second class constraints $\left(p_{\lambda}, p_{\mathbf{n}}, \mathcal{C}_{\lambda}, \mathcal{C}_{\mathbf{n}}\right)$. We can again gauge fix the scale symmetry as in section 2.1 in order to obtain the Hamiltonian constraint in the form

$$
\mathcal{H}_{T}=\mathcal{H}_{T}^{\mathrm{GR}}+\mathcal{H}_{T}^{u}
$$

where the contribution of the vector field is given as

$$
\begin{aligned}
\mathcal{H}_{T}^{u}= & \frac{1}{2 \mu^{2} \sqrt{h}} p^{i} h_{i j} p^{j}+\sqrt{1+u_{i} h^{i j} u_{j}} D_{i} p^{i} \\
& +\frac{\mu^{2}}{4} \sqrt{h} h^{i k} h^{j l}\left(D_{i} u_{j}-D_{j} u_{i}\right)\left(D_{k} u_{l}-D_{l} u_{k}\right) .
\end{aligned}
$$

From (4.19) or (4.21) we can read following important information. Three physical degrees of freedom are carried in the vector field and two in the metric. The Hamiltonian constraint $\mathcal{H}_{T}$ depends on the momenta $p^{i}$ quadratically and due to the fact that we have chosen sign in front of the square root (4.17) we also see that the vector part is positive definite and hence bounded from below. In other words there is no sign of the ghost instability. In summary, the vector form of the mimetic model seems to be very promising model of the dark energy and deserves to be elaborated further.

\section{Mimetic tensor-vector-scalar gravity}

We propose a tensor-vector-scalar theory of gravity that is a generalization of the theories of mimetic dark matter $[1,2]$. The gravitational action of this theory includes both a vector field $u_{\mu}$ and a scalar field $\phi$. Several different tensor-vector-scalar theories of gravity have 
been proposed in order to address the problem of dark matter, most notably the theories proposed by Bekenstein [10] and Moffat [11]. Our proposal differs from all the previous tensor-vector-scalar theories.

The physical metric is defined to have the form

$$
g_{\mu \nu}^{\text {phys }}=-f(\phi)\left(g^{\alpha \beta} u_{\alpha} u_{\beta}\right) g_{\mu \nu} \equiv \Phi^{2} g_{\mu \nu}
$$

where $f(\phi)$ is some (dimensionless) nonnegative function of $\phi$, e.g., $f(\phi) \propto \phi^{2}$. The physical metric is again invariant under the conformal transformation of the metric $g_{\mu \nu}$. The standard kinetic term and an optional potential term for the scalar field $\phi$ are included into the action (4.2) so that the total gravitational action has the form

$$
\begin{aligned}
S\left[g_{\mu \nu}^{\text {phys }}, u_{\mu}, \phi\right]= & \int d^{4} x \sqrt{-g^{\text {phys }}}\left[\frac{1}{2} R\left(g_{\mu \nu}^{\text {phys }}\right)-\frac{\mu^{2}}{4} g_{\text {phys }}^{\mu \alpha} g_{\text {phys }}^{\nu \beta} F_{\mu \nu} F_{\alpha \beta}\right. \\
& \left.-\frac{1}{2} g_{\text {phys }}^{\mu \nu} \nabla_{\mu}^{\text {phys }} \phi \nabla_{\nu}^{\text {phys }} \phi-V(\phi)+L\left(g_{\mu \nu}^{\text {phys }}, \chi, \partial \chi, u_{\mu}, \phi\right)\right] .
\end{aligned}
$$

The Lagrangian $L\left(g_{\mu \nu}^{\text {phys }}, \chi, \partial \chi, u_{\mu}, \phi\right)$ represents matter and its interaction with the gravitational fields. Variation of the action with respect to the physical metric $g_{\mu \nu}^{\text {phys }}$ implies the standard gravitational field equations. The variation is given as

$$
\begin{aligned}
\delta_{g_{\mu \nu}^{\text {phys }}} S= & \frac{1}{2} \int d^{4} x \sqrt{-g^{\text {phys }}}\left(-G_{\text {phys }}^{\mu \nu}+T_{F}^{\mu \nu}+T_{\phi}^{\mu \nu}+T^{\mu \nu}\right) \delta g_{\mu \nu}^{\text {phys }} \\
& -\oint d^{3} x \sqrt{\left|\gamma^{\text {phys }}\right|} \delta K^{\text {phys }},
\end{aligned}
$$

where

$$
\begin{aligned}
G_{\mathrm{phys}}^{\mu \nu} & =R^{\mu \nu}\left(g_{\mu \nu}^{\mathrm{phys}}\right)-\frac{1}{2} g_{\mathrm{phys}}^{\mu \nu} R\left(g_{\mu \nu}^{\mathrm{phys}}\right), \\
T_{F}^{\mu \nu} & =\mu^{2}\left(F^{\mu \alpha} F_{\alpha}^{\nu}-\frac{1}{4} g_{\mathrm{phys}}^{\mu \nu} F_{\alpha \beta} F^{\alpha \beta}\right), \\
T_{\phi}^{\mu \nu} & =\nabla_{\mathrm{phys}}^{\mu} \phi \nabla_{\mathrm{phys}}^{\nu} \phi-g_{\mathrm{phys}}^{\mu \nu}\left(\frac{1}{2} \nabla_{\alpha}^{\mathrm{phys}} \phi \nabla_{\mathrm{phys}}^{\alpha} \phi+V(\phi)\right)
\end{aligned}
$$

and

$$
T_{\text {phys }}^{\mu \nu}=\frac{2}{\sqrt{-g^{\text {phys }}}} \frac{\delta}{\delta g_{\mu \nu}^{\text {phys }}} \int d^{4} x \sqrt{-g^{\text {phys }}} L\left(g_{\mu \nu}^{\text {phys }}, \chi, \partial \chi, u_{\mu}, \phi\right) .
$$

Note that above everything is written in the physical frame, e.g., $F^{\alpha \beta}=g_{\text {phys }}^{\alpha \mu} g_{\text {phys }}^{\beta \nu} F_{\mu \nu}$. The second integral in (5.3) is taken over the boundary of spacetime with $\gamma^{\text {phys }}$ and $K^{\text {phys }}$ being the determinant of the induced metric and the trace of the extrinsic curvature on the boundary, respectively. This surface contribution could be canceled by adding an appropriate boundary term into the action. 
When the action is viewed as a functional of $g_{\mu \nu}, u_{\mu}, \phi$ and the matter fields, the variation of the physical metric reads as

$$
\begin{aligned}
\delta g_{\mu \nu}^{\text {phys }}\left(g_{\mu \nu}, u_{\mu}, \phi\right)= & \Phi^{2} \delta g_{\mu \nu}+g_{\mu \nu} \delta \Phi^{2} \\
= & \Phi^{2} \delta g_{\mu \nu}+g_{\mu \nu} f(\phi) g^{\alpha \rho} g^{\alpha \sigma} u_{\rho} u_{\sigma} \delta g_{\alpha \beta} \\
& -2 g_{\mu \nu} f(\phi) g^{\alpha \beta} u_{\alpha} \delta u_{\beta}-g_{\mu \nu} f^{\prime}(\phi)\left(g^{\alpha \beta} u_{\alpha} u_{\beta}\right) \delta \phi \\
= & \Phi^{2}\left(\delta_{\mu}^{\alpha} \delta_{\nu}^{\beta}+g_{\mu \nu}^{\text {phys }} f(\phi) g_{\text {phys }}^{\alpha \rho} g_{\text {phys }}^{\alpha \sigma} u_{\rho} u_{\sigma}\right) \delta g_{\alpha \beta} \\
& -2 g_{\mu \nu}^{\text {phys }} f(\phi) g_{\text {phys }}^{\alpha \beta} u_{\alpha} \delta u_{\beta}-g_{\mu \nu}^{\text {phys }} f^{\prime}(\phi)\left(g_{\text {phys }}^{\alpha \beta} u_{\alpha} u_{\beta}\right) \delta \phi .
\end{aligned}
$$

Hence we obtain the following gravitational field equations:

$$
\begin{array}{r}
G_{\text {phys }}^{\mu \nu}=T_{\text {phys }}^{\mu \nu}+\varepsilon u^{\mu} u^{\nu}+T_{F}^{\mu \nu}+T_{\phi}^{\mu \nu}, \\
\mu^{2} \nabla_{\nu}^{\text {phys }} F^{\nu \mu}-\varepsilon u^{\mu}+\frac{\partial L}{\partial u_{\mu}}=0, \\
\nabla_{\mu}^{\text {phys }} \nabla_{\text {phys }}^{\mu} \phi-\varepsilon u_{\mu} u^{\mu} \frac{f^{\prime}(\phi)}{2 f(\phi)}-V^{\prime}(\phi)+\frac{\partial L}{\partial \phi}=0,
\end{array}
$$

where $u^{\mu}=g_{\text {phys }}^{\mu \nu} u_{\nu}$ is the velocity of the mimetic dust, whose energy density is given as

$$
\varepsilon=f(\phi) g_{\mu \nu}^{\text {phys }}\left(T_{\text {phys }}^{\mu \nu}+T_{\phi}^{\mu \nu}-G_{\text {phys }}^{\mu \nu}\right) .
$$

The metric $g_{\mu \nu}$ enters the field equations only through the physical metric (5.1), while the vector field and the scalar field appear also explicitly. The main difference compared to the vector field model [2] is the presence of the extra scalar field $\phi$ in the energy of the dust (5.12). The fields $\phi, u_{\mu}, g_{\mu \nu}$ are also coupled to each other in a rather intricate way, even in the absence of matter fields. These are the characteristics that make $\phi$ differ from a regular scalar field that can be included into the Lagrangian of matter.

\subsection{Hamiltonian formulation}

We again introduce $\Phi$ as an independent variable, rewriting the action as

$$
\begin{aligned}
S\left[g_{\mu \nu}, \Phi, \lambda, u_{\mu}, \phi\right]= & \int d^{4} x \sqrt{-g}\left[\frac{1}{2} \Phi^{2} R\left(g_{\mu \nu}\right)+3 g^{\mu \nu} \nabla_{\mu} \Phi \nabla_{\nu} \Phi\right. \\
& +\frac{1}{2} \lambda\left(\Phi^{2}+f(\phi) g^{\mu \nu} u_{\mu} u_{\nu}\right)-\frac{\mu^{2}}{4} g^{\mu \alpha} g^{\nu \beta} F_{\mu \nu} F_{\alpha \beta} \\
& \left.-\frac{1}{2} \Phi^{2} g^{\mu \nu} \nabla_{\mu} \phi \nabla_{\nu} \phi-\Phi^{4} V(\phi)\right] .
\end{aligned}
$$

We again ignore the boundary terms since we are interested in the local (propagating) degrees of freedom, rather than the invariant surface energy.

In the ADM description, the scalar field action has the form

$$
S_{\phi}=\frac{1}{2} \int d t d^{3} x \sqrt{h} N \Phi^{2}\left[\left(\nabla_{n} \phi\right)^{2}-h^{i j} \partial_{i} \phi \partial_{j} \phi-\Phi^{4} V(\phi)\right],
$$


where

$$
\nabla_{n} \phi=\frac{1}{N}\left(\partial_{t} \phi-N^{i} \partial_{i} \phi\right) .
$$

The canonical momentum conjugate to $\phi$ is defined as

$$
p_{\phi}=\sqrt{h} \Phi^{2} \nabla_{n} \phi .
$$

The other canonical momenta - as well as all the primary constraints - are the same as for the vector field model in section 4 . We obtain the Hamiltonian as

$$
\begin{aligned}
H= & \int d^{3} \mathbf{x}\left(N \mathcal{H}_{T}+N^{i} \mathcal{H}_{i}+v_{\mathcal{D}} \mathcal{D}+v_{N} \pi_{N}+v^{i} \pi_{i}+v_{\lambda} p_{\lambda}+v_{\mathbf{n}} p_{\mathbf{n}}\right) \\
\mathcal{H}_{T}= & \frac{2}{\sqrt{h} \Phi^{2}} \pi^{i j} \mathcal{G}_{i j k l} \pi^{k l}-\frac{1}{2} \sqrt{h} R \Phi^{2}+\partial_{i}\left[\sqrt{h} h^{i j} \partial_{j} \Phi^{2}\right] \\
& -3 \sqrt{h} h^{i j} \partial_{i} \Phi \partial_{j} \Phi-\frac{1}{2} \sqrt{h} \lambda\left(\Phi^{2}+f(\phi) u_{i} h^{i j} u_{j}-f(\phi) u_{\mathbf{n}}^{2}\right) \\
& +\frac{1}{2 \mu^{2} \sqrt{h}} p^{i} h_{i j} p^{j}+\frac{\mu^{2}}{4} \sqrt{h} h^{i k} h^{j l}\left(D_{i} u_{j}-D_{j} u_{i}\right)\left(D_{k} u_{l}-D_{l} u_{k}\right)-u_{\mathbf{n}} D_{i} p^{i} \\
& +\frac{p_{\phi}^{2}}{2 \sqrt{h} \Phi^{2}}+\frac{1}{2} \sqrt{h} \Phi^{2} h^{i j} \partial_{i} \phi \partial_{j} \phi+\Phi^{4} V(\phi), \\
\mathcal{H}_{i}= & p_{\Phi} \partial_{i} \Phi-2 h_{i k} D_{j} \pi^{k j}+\partial_{i} u_{j} p^{j}-\partial_{j}\left(u_{i} p^{j}\right)+p_{\phi} \partial_{i} \phi .
\end{aligned}
$$

The secondary constraints that ensure the preservation of $p_{\lambda} \approx 0$ and $p_{\mathbf{n}} \approx 0$ are defined as

$$
\mathcal{C}_{\lambda}=\sqrt{h}\left(\Phi^{2}+f(\phi) u_{i} h^{i j} u_{j}-f(\phi) u_{\mathbf{n}}^{2}\right) \approx 0
$$

and

$$
\mathcal{C}_{\mathbf{n}}=-\sqrt{h} \lambda f(\phi) u_{\mathbf{n}}+D_{i} p^{i} \approx 0,
$$

respectively. These are second-class constraints. The constraint $\mathcal{C}_{\lambda}=0$ can be solved for

$$
u_{\mathbf{n}}=-\sqrt{\frac{\Phi^{2}}{f(\phi)}+u_{i} h^{i j} u_{j}},
$$

assuming $f(\phi)>0$. Then $\lambda$ is fixed by the constraint $\mathcal{C}_{\mathbf{n}}=0$. The Hamiltonian constraint is given as

$$
\begin{aligned}
\mathcal{H}_{T}= & \frac{2}{\sqrt{h} \Phi^{2}} \pi^{i j} \mathcal{G}_{i j k l} \pi^{k l}-\frac{1}{2} \sqrt{h} R \Phi^{2}+\partial_{i}\left[\sqrt{h} h^{i j} \partial_{j} \Phi^{2}\right] \\
& -3 \sqrt{h} h^{i j} \partial_{i} \Phi \partial_{j} \Phi \\
& +\frac{1}{2 \mu^{2} \sqrt{h}} p^{i} h_{i j} p^{j}+\sqrt{\frac{\Phi^{2}}{f(\phi)}+u_{i} h^{i j} u_{j} D_{i} p^{i}} \\
& +\frac{\mu^{2}}{4} \sqrt{h} h^{i k} h^{j l}\left(D_{i} u_{j}-D_{j} u_{i}\right)\left(D_{k} u_{l}-D_{l} u_{k}\right) \\
& +\frac{p_{\phi}^{2}}{2 \sqrt{h} \Phi^{2}}+\frac{1}{2} \sqrt{h} \Phi^{2} h^{i j} \partial_{i} \phi \partial_{j} \phi+\Phi^{4} V(\phi) .
\end{aligned}
$$


There exist six physical degrees of freedom, since we have seventeen pairs of canonical variables, nine first class constraints $\left(\mathcal{H}_{T}, \mathcal{H}_{i}, \pi_{N}, \pi_{i}, \mathcal{D}\right)$ and four second class constraints $\left(p_{\lambda}, p_{\mathbf{n}}, \mathcal{C}_{\lambda}, \mathcal{C}_{\mathbf{n}}\right)$. Furthermore, one can fix the conformal gauge as in (2.45), obtaining a true tensor-vector-scalar theory without auxiliary fields, whose Hamiltonian constraint is given as

$$
\mathcal{H}_{T}=\mathcal{H}_{T}^{\mathrm{GR}}+\mathcal{H}_{T}^{u, \phi},
$$

where the contribution of the vector and scalar fields is given as

$$
\begin{aligned}
\mathcal{H}_{T}^{u, \phi}= & \frac{1}{2 \mu^{2} \sqrt{h}} p^{i} h_{i j} p^{j}+\sqrt{\frac{1}{f(\phi)}+u_{i} h^{i j} u_{j} D_{i} p^{i}} \\
& +\frac{\mu^{2}}{4} \sqrt{h} h^{i k} h^{j l}\left(D_{i} u_{j}-D_{j} u_{i}\right)\left(D_{k} u_{l}-D_{l} u_{k}\right) \\
& +\frac{p_{\phi}^{2}}{2 \sqrt{h}}+\frac{1}{2} \sqrt{h} h^{i j} \partial_{i} \phi \partial_{j} \phi+V(\phi) .
\end{aligned}
$$

Alternatively, one could fix the conformal gauge, for example, as $\Phi^{2}=f(\phi)$, i.e., in a similar way as in the vector field model, $-g^{\mu \nu} u_{\mu} u_{\nu}=1$.

The Hamiltonian (5.21) or (5.22) has quite similar characteristics compared to the vector case in section 4 . Three physical degrees of freedom are carried in the vector field, one in the scalar field $\phi$, and two in the metric. All the fields have well-defined kinetic terms. Presuming the potential $V(\phi)$ is stable, the addition of the extra field $\phi$ does not imply any evident problems. The scalar mode $\phi$ is coupled to both the tensor and vector modes, as well as to the scalar $\Phi$.

\section{Conclusions}

We have studied the potential existence of ghost instability in the recently proposed gravitational theories featuring mimetic dark matter. The original mimetic dark matter model [1] - that is a conformal extension of Einstein's gravity containing an extra scalar field that can mimic dusty dark matter - obtains a Hamiltonian that is linear in the momentum $p_{\phi}$ conjugate to the scalar field $\phi$. The Lagrange multiplier $\lambda$ describes the rest mass density of the mimetic dust, which is required to be positive on the initial Cauchy surface. It is solved in terms of $p_{\phi}$ and the other canonical variables in (2.31). If we require that $\lambda>0$ at all times, the Hamiltonian has a structure that is known to describe dust [25]. However, for certain type of initial configurations, the requirement $\lambda>0$ is inconsistent with the dynamics governed by the equations of motion. We indeed argue that there exist configurations for which the momentum $p_{\phi}$ can evolve to zero and then become negative. In those cases, the density of the mimetic dust becomes negative under time evolution, which means that the system becomes unstable. Actually, the requirement $\lambda>0$ appears to be equivalent to choosing the initial configurations that do no cross the surface $p_{\phi}=0$ under time evolution. Only those configurations can be used to describe physical systems. Lastly, in section 3, the formulation of the theory in the Einstein frame is achieved by gauge fixing the conformal symmetry of the original action. 
The alternative model with an extra vector field [2] is shown to be well defined from the Hamiltonian point of view. This model was shown in [2] to be able to mimic both potential and rotational flows of a pressureless perfect fluid. Finally, we have presented a mimetic tensor-vector-scalar theory of gravity which contains both a scalar field and a vector field. It is shown to possess a healthy canonical structure that is free of ghost degrees of freedom. The inclusion of the scalar field further generalizes the dynamics, letting both the scalar and vector degrees of freedom contribute to the mimetic matter.

Phenomenological implications of the tensor-vector-scalar gravity proposed in section 5 should be studied carefully. The inclusion of a scalar field in addition to a vector field certainly has a significant effect compared to the previous models [1-3]. Implications to cosmology and structure formation are among the main interests. The scalar field $\phi$ could play a role similar to a conventional inflaton field. In that case, does its unusual coupling to $\Phi$ imply any advantage over a conventional minimally coupled inflaton field, or is the inclusion of $\phi$ into (5.1) just an unnecessary complication? Likewise, for a different potential and couplings, the scalar field $\phi$ could produce the late-time acceleration, mimicking dark energy.

\section{Acknowledgments}

We thank Andrei Barvinsky and Viatcheslav Mukhanov for several clarifying discussions. The support of the Academy of Finland under the Project Nos. 136539 and 272919, as well as of the Magnus Ehrnrooth Foundation, is gratefully acknowledged. The work of J.K. was supported by the Grant Agency of the Czech Republic under the grant P201/12/G028. The work of M.O. was supported by the Jenny and Antti Wihuri Foundation.

Open Access. This article is distributed under the terms of the Creative Commons Attribution License (CC-BY 4.0), which permits any use, distribution and reproduction in any medium, provided the original author(s) and source are credited.

\section{References}

[1] A.H. Chamseddine and V. Mukhanov, Mimetic Dark Matter, JHEP 11 (2013) 135 [arXiv: 1308.5410] [INSPIRE].

[2] A.O. Barvinsky, Dark matter as a ghost free conformal extension of Einstein theory, JCAP 01 (2014) 014 [arXiv: 1311.3111] [INSPIRE].

[3] A.H. Chamseddine, V. Mukhanov and A. Vikman, Cosmology with Mimetic Matter, JCAP 06 (2014) 017 [arXiv: 1403.3961] [INSPIRE].

[4] G. Nordström, Zur Theorie der Gravitation vom Standpunkt des Relativitätsprinzips, Ann. Phys. 347 (1913) 533.

[5] A. Einstein and A. D. Fokker, Die Nordströmsche Gravitationstheorie vom Standpunkt des absoluten Differentialkalküls, Ann. Phys. 349 (1914) 321.

[6] E.A. Lim, I. Sawicki and A. Vikman, Dust of Dark Energy, JCAP 05 (2010) 012 [arXiv: 1003.5751] [INSPIRE]. 
[7] C. Gao, Y. Gong, X. Wang and X. Chen, Cosmological models with Lagrange Multiplier Field, Phys. Lett. B 702 (2011) 107 [arXiv: 1003.6056] [INSPIRE].

[8] S. Capozziello, J. Matsumoto, S. Nojiri and S.D. Odintsov, Dark energy from modified gravity with Lagrange multipliers, Phys. Lett. B 693 (2010) 198 [arXiv:1004.3691] [INSPIRE].

[9] J. Kluson, Hamiltonian Analysis of Lagrange Multiplier Modified Gravity, Class. Quant. Grav. 28 (2011) 125025 [arXiv: 1009.6067] [INSPIRE].

[10] J.D. Bekenstein, Relativistic gravitation theory for the MOND paradigm, Phys. Rev. D 70 (2004) 083509 [Erratum ibid. D 71 (2005) 069901] [astro-ph/0403694] [INSPIRE].

[11] J.W. Moffat, Scalar-tensor-vector gravity theory, JCAP 03 (2006) 004 [gr-qc/0506021] [INSPIRE].

[12] M. Chaichian, J. Kluson, M. Oksanen and A. Tureanu, Can TeVeS be a viable theory of gravity?, Phys. Lett. B 735 (2014) 322 [arXiv:1402.4696] [INSPIRE].

[13] V. Faraoni, E. Gunzig and P. Nardone, Conformal transformations in classical gravitational theories and in cosmology, Fund. Cosmic Phys. 20 (1999) 121 [gr-qc/9811047] [InSPIRE].

[14] R.L. Arnowitt, S. Deser and C.W. Misner, The dynamics of general relativity, Gen. Rel. Grav. 40 (2008) 1997 [gr-qc/0405109] [INSPIRE].

[15] E. Gourgoulhon, $3+1$ formalism and bases of numerical relativity, gr-qc/0703035 [INSPIRE].

[16] R. Schon and S.-T. Yau, Proof of the positive mass theorem. II, Commun. Math. Phys. 79 (1981) 231 [InSPIRE].

[17] E. Witten, A simple proof of the positive energy theorem, Commun. Math. Phys. 80 (1981) 381 [InSPIRE].

[18] R.P. Woodard, Avoiding Dark Energy with 1/R Modifications of Gravity, Lect. Notes Phys. 720 (2007) 403, [astro-ph/0601672].

[19] J. Klusoň, M. Oksanen and A. Tureanu, Hamiltonian analysis of curvature-squared gravity with or without conformal invariance, Phys. Rev. D 89 (2014) 064043 [arXiv:1311.4141] [INSPIRE].

[20] M. Chaichian, I. Merches and A. Tureanu, Mechanics: An Intensive Course, Springer-Verlag, (2012), pg. 278.

[21] G. 't Hooft, Quantum mechanics and determinism, hep-th/0105105 [INSPIRE].

[22] G. 't Hooft, Quantum gravity as a dissipative deterministic system, Class. Quant. Grav. 16 (1999) 3263 [gr-qc/9903084] [INSPIRE].

[23] M. Blasone, P. Jizba and F. Scardigli, Can quantum mechanics be an emergent phenomenon?, J. Phys. Conf. Ser. 174 (2009) 012034 [arXiv:0901.3907] [InSPIRE].

[24] M. Blasone, P. Jizba and H. Kleinert, Path integral approach to 't Hooft's derivation of quantum from classical physics, Phys. Rev. A 71 (2005) 052507 [quant-ph/0409021] [INSPIRE].

[25] J.D. Brown and K.V. Kuchar, Dust as a standard of space and time in canonical quantum gravity, Phys. Rev. D 51 (1995) 5600 [gr-qc/9409001] [INSPIRE].

[26] A. Golovnev, On the recently proposed Mimetic Dark Matter, Phys. Lett. B 728 (2014) 39 [arXiv: 1310.2790] [INSPIRE]. 